\title{
SOME characteristic properties of analytical method of magnetic control of ferroimpurities in various primary and technological media
}

\author{
Anna A. Sandulyak ${ }^{1 a}$, Alexander V. Sandulyak ${ }^{1}$, Hasan Bulut ${ }^{2}$, Haci Mehmet Baskonus ${ }^{3}$, Maria N. Polismakova ${ }^{1}$, \\ Darya A. Sandulyak ${ }^{1}$ \\ ${ }^{1}$ Moscow Technological University, 107996 Moscow, Stromynka, 20, RF \\ 2 Department of Mathematics, Firat University, 23119, Elazig, Turkey \\ ${ }^{3}$ Faculty of Engineering, Tunceli University, 62100, Tunceli, Turkey
}

\begin{abstract}
The paper demonstrates that when defining the content of ferroimpurities in the analyzed media by magnetic control based on measuring the ferroimpurities masses $m$, which are extracted from the media sample step-by-step, it is crucial to define a functional view of the obtained dependence $m$ on the operation order number $n$. It allows receiving additional forecast data $\mathrm{m}$ by extrapolating this dependence. With corresponding calculations being done, it enables determining the sought total mass of ferroimpurities (including their residual mass). We consider alternate solutions of practical tasks which yield magnetic control results in case of linear and piecewise-linear views (in semi-logarithmic coordinates) of the control mass-operational characteristics.
\end{abstract}

\section{Introduction}

Many industries face a challenge of inevitable presence of ferroimpurities in primary and technological media. Deteriorating the quality of these media and thus impoverishing the quality of the final product, such impurities can also seriously destabilize the technological process and the performance of manufacturing equipment. Therefore, it is important to ensure valid control of ferroimpurities content. The classification of a medium by normative quality targets greatly depends on solution of this problem which also influences the timely decisions and efficient removal of ferroimpurities (e.g. with the help of magnetic separators [1-6]). Besides, it is important for modeling of the process of magnetic impact on ferroimpurities [7-13].

It is common practice for control methods to use a test-method control based on magnetic extraction of ferroimpurities from the sample of the medium analysed (magnetophoresis), measuring their masses and further evaluation of their mass share (concentration). For a more complete removal of ferroimpurities, the magnetophoretic operations are as a rule performed several times, recurrently, cyclically with determining the accumulated mass of the ferroimpurities re- moved from the sample. In such a case, there is assigned some recommended 'sufficient' number $\mathrm{n}$ of such operations.

However, we believe that such recommendations are not to be taken for granted, but they are to be discussed. The matter is, after performing even an increased $n$ number of magnetophoretic operations, a sample of a medium still contains a residual (notremoved) mass of ferroimpurities. As far as the $n$ number increases, the value of this mass (from general physical concepts it is always present) surely reduces (and the quality of control improves), but we have to admit it reduces asymptotically nearing zero only when the number of operations is infinitely unlimited.

Yet such an approach stipulating multiple (reasonably limited by practically substantiated number of operations) magnetic removal of ferroimpurities from the sample of the medium analyzed is justified. Herewith, it becomes effective to the extent required if it is followed by obtaining an experimental dependence (decreasing) of the measured operational masses $\mathrm{m}$ of removed ferroparticles on the sequence order number $\mathrm{n}$ of the operation. It is similar to the process when we study the nature of reducing ferroparticles concentration progressively as wefurther

\footnotetext{
a Corresponding author: anna.sandulyak@mail.ru
} 
execute multiple magnetophoretic operations for removing ferroparticles from liquids [14]. This particular dependence can serve as a basis for substantiating and execution of the experimental and calculating method of ferroimpurities magnetic control.

\section{Main results and their discussion}

Obtaining experimental dependence of operational masses $m$ of extracted ferroimpurities on the operation sequence number $n$ is one of the first steps in the experimental and calculating method of ferroimpurities magnetic control considered here. The second step required for the method is identifying the functional view of dependence $m$ on $n$. Thus, its functional legalization allows for objective extrapolation up to any value $n$, no matter how big it is, and consequently, it affords obtaining values $m$ lying beyond actual experiment by calculating [15].

Many experimental dependences of $m$ on $n$, in particular, the ones depicted in Fig. $1 a-4 a$ (obtained during multiple operations of magnetic separation of ferroimpurities from flour, quartz sand, petrol,

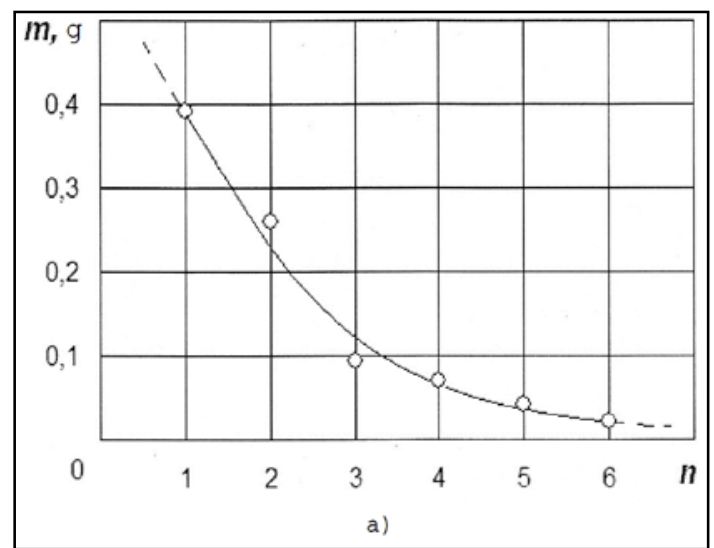

Figure 1. Experimental data of ferroimpurities masses removed from flour after each magnetophoretic operation -

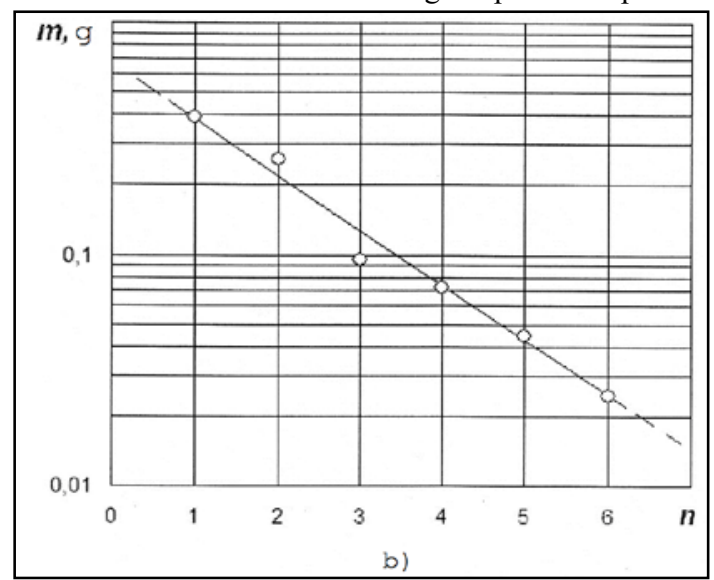

in common (a) and semilogarithmic (b) coordinates.
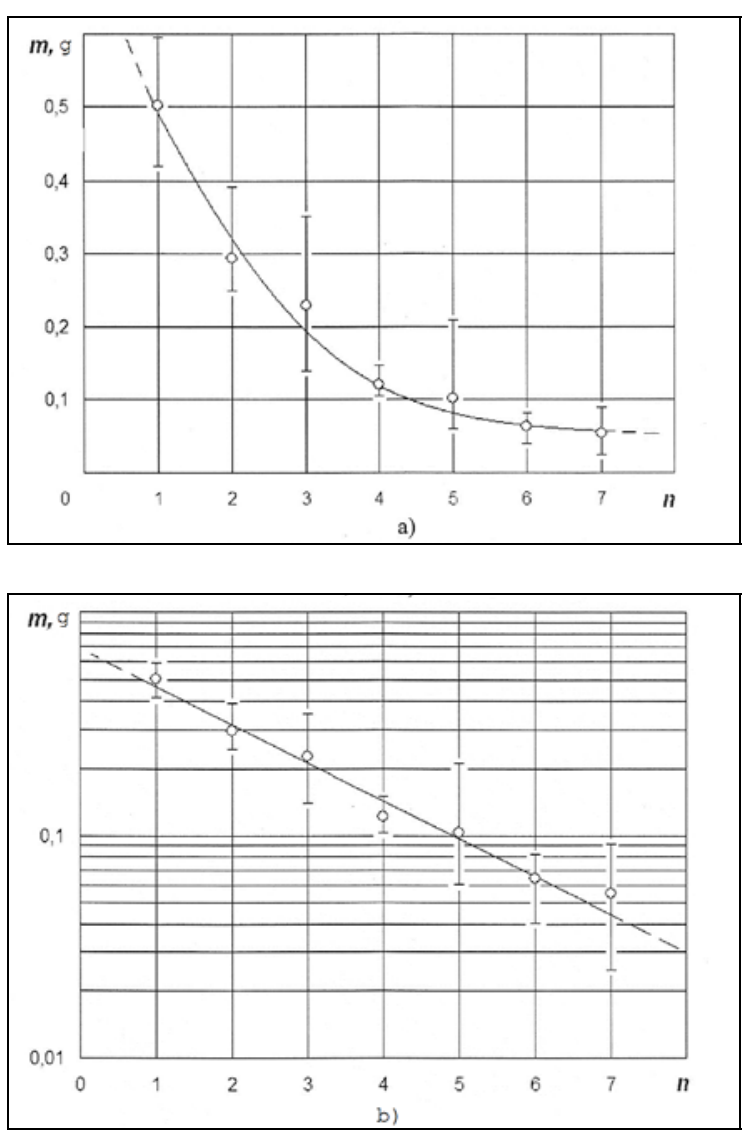

Figure 2. The same data as in Fig. 1 but for quartz sand.

engine oil), linearize quite well in semilogarithmic coordinates (Fig. $1 b-4 b$ ). This proves the regularity of operational masses exponential reduction:

$$
m=A \cdot \exp (-k \cdot n),
$$

Summing up the experimental data (Fig. 1 - 4), expression (1) actually provides an extrapolational forecast of values of further operational masses. At that for specific cases of ferroimpurities magnetic control, parameter A in (1) is determined graphically as a fictitious starting $(n=0)$ ordinate which is 'linearized' in logarithmic coordinates of dependence $\mathrm{m}$ on $\mathrm{n}($ Fig. $1 b-4 b)$. Another parameter in (1) is $\mathrm{k}$, which is defined by inverse recalculation with an arbitrary pair (Fig. $1-4$ ) of corresponding values of $n$ and $m=m n$, i.e. $k=[-\ln (m n / A)] / n$. Besides, it is also possible to employ Excel spreadsheet to develop expression (1) with its inherent particular values of parameters $\mathrm{A}$ and $\mathrm{k}$ during ferroimpurities magnetic control in some medium.

After determining expression (1) we can have at our disposal the data on total aggregate value of ferroimpurities masses, including residual (not extracted) masses as well. 


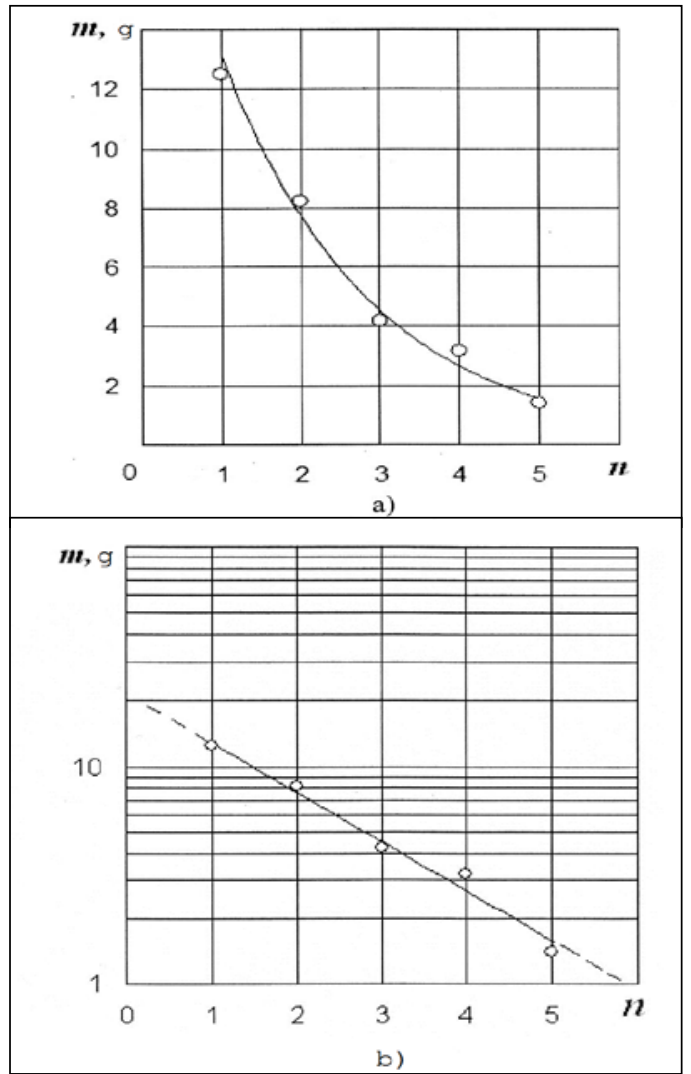

Figure 3. The same data as in Fig. 1 but for engine oil.

At the first glance, it may seem that we would need to sum up as many as possible members of discrete dependence (1), up to $n \rightarrow \infty$.

Having said that, we still claim this summing up is easily done if we take into consideration that discrete dependence (1) is a quantitative series represented as a decreasing geometric progression. Consequently, with the known first member of this progression $\mathrm{ml}=\mathrm{A} \cdot \exp (-\mathrm{k} \cdot 1)$ and its ratio $\mathrm{q}=\exp (-\mathrm{k})$ the mass of ferroimpurities in the medium analyzed is determined as a total potential mass of residue $\mathrm{ml} \ldots \infty$, which could be obtained with virtually unlimited number of operations $(\mathrm{n} \rightarrow \infty)$, i.e.

$$
m_{1 \ldots \infty}=\sum_{n=1}^{\infty} m_{n}=\frac{m_{1}}{1-q}=\frac{A}{\exp k-1} .
$$

Further, with known values of volume $V$ and mass $\mathrm{M}$ of the analysed medium sample, we can find true values of volume and/or mass-concentrations $C_{V}$ and $C_{M}$ respectively: $\mathrm{cV}=\mathrm{m} 1 \ldots \infty / \mathrm{V}, \mathrm{cM}=\mathrm{m} 1 \ldots \infty / \mathrm{M}$.

In case when a limited number of operations $n$ is performed, employing the same discrete dependence (1) we can find the mass of the ferroimpurities removed from the sample analysed $\mathrm{m} 1$...nas a sum of the limited number of geometric progression members:

$$
m_{1 \ldots n}=\sum_{n=1}^{n} m_{n}=\frac{m_{1}-m_{n} \cdot q}{1-q}=A \frac{1-\exp (-k \cdot n)}{\exp k-1}
$$

In which case, with the account for (1) and (3) we can assess the corresponding error $\varepsilon 1 \ldots \mathrm{n}=(\mathrm{m} 1 \ldots \infty$ $\mathrm{m} 1 \ldots \mathrm{n}) / \mathrm{m} 1 \ldots \infty=\exp (-\mathrm{k} \cdot \mathrm{n})(\mathrm{x} 100 \%)$ for the case when we take into consideration only the data for some number $\mathrm{n}$ of actually performed magnetophoretic operations.

It is noteworthy, that the afore-described methodology of magnetic control (its calculating part) is true not for all media. Thus, similar experiments on polyoperational extraction of ferroimpurities from a sample of other media, e.g. feldspar, demonstrated the following. The obtained mass-operational dependence (Fig. 5a) was at first sight a decreasing and asymptomatically nearing zero one, but as a whole, it was functionally different from the basic dependence (1). We also proved it true that in test semi logarithmic coordinates (Fig. 5b) this dependence could not be fully linearized. However, it can be partially linearized as two sections with clearly expressed 'kink', which actually suggests that the described experimental and calculating method of magnetic control can be possibly adapted to working with such specific media.
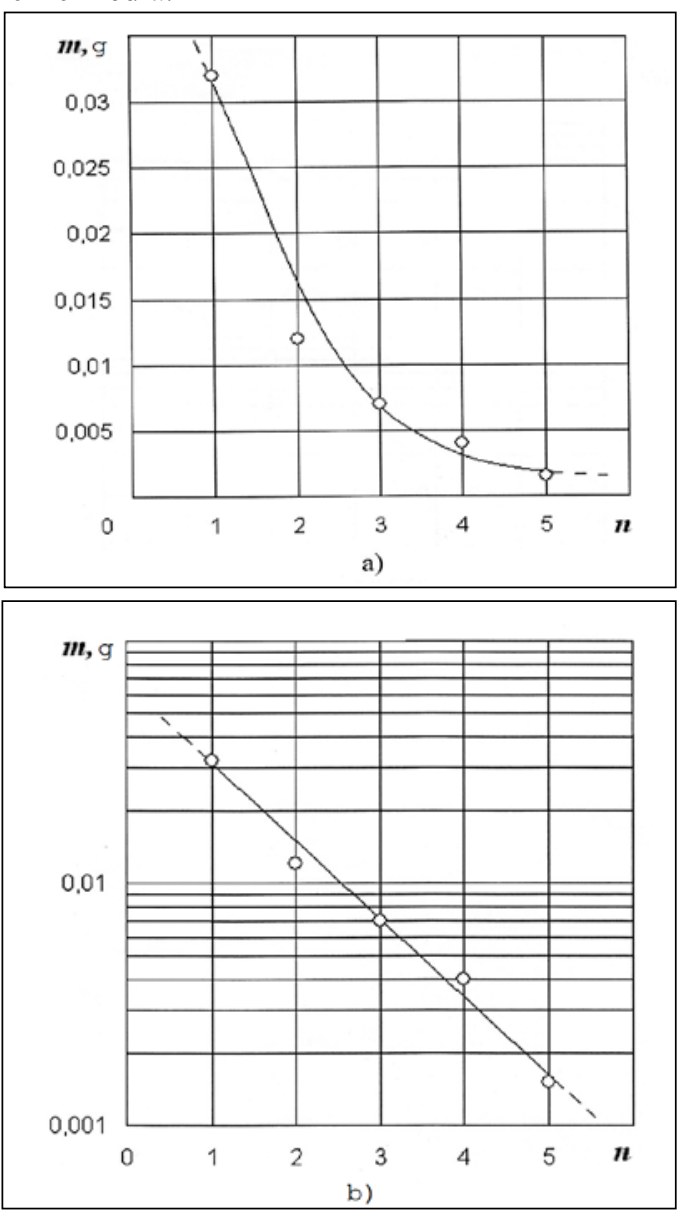

Figure 4. The same data as in Fig. 1 but for petrol. 

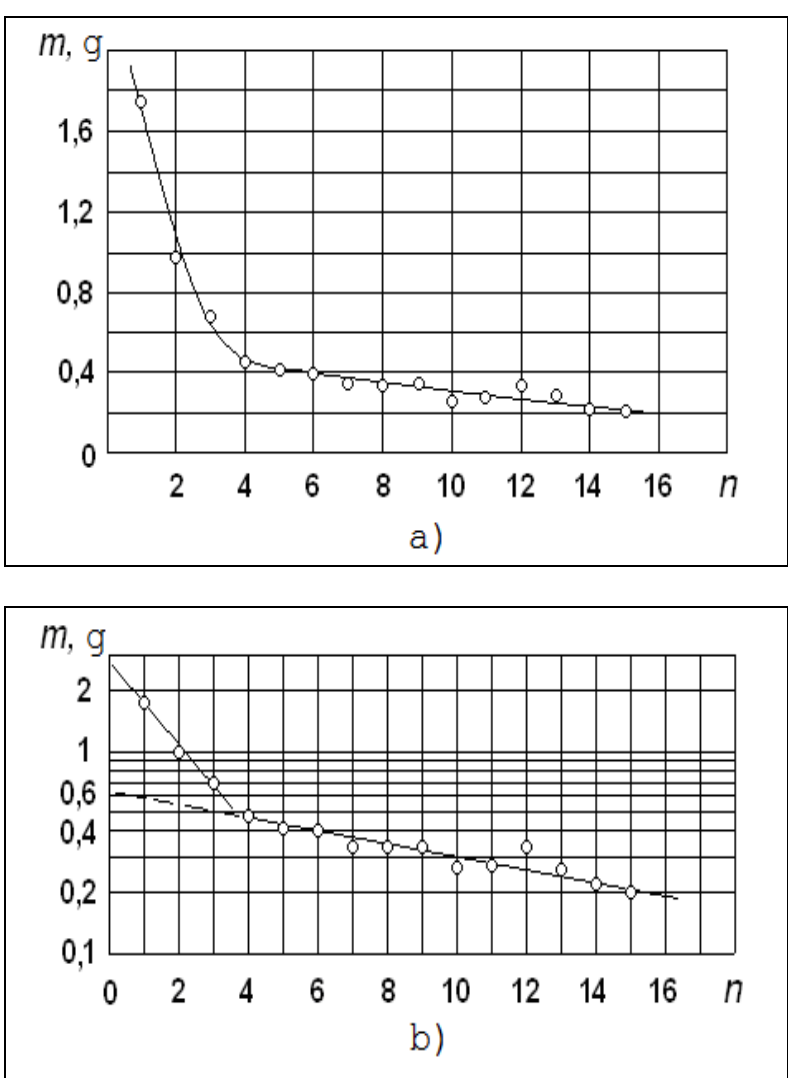

Figure 5. The same data as in Fig. 1 but for feldspar.

Essentially, such adaptation comes down to the following. Total mass $m_{1 \ldots \infty}$ of ferroimpurities in the sample analysed is obviously (Fig. $5 b$ ):

$$
m_{1 \ldots \infty}=m_{1 \ldots n}+m_{(n+1) \ldots \infty} \text {. }
$$

Providing the 'kink' of $m, n$-dependence (Fig. 6b) falls on some value $n$, as a rule, a small one (Fig.5b, $n=4$ ), then value $m_{1 \ldots n}$ (for the first steeply descending initial section) can be easily determined immediately by a simple summation of factual data of operational masses, i.e. $m_{1 \ldots n}=m_{1}+m_{2}+\ldots+m_{n}$. Whereas when calculating mass $m(n+1) \ldots \infty$ (for the next, second section, including the data of extrapolated operations) we should subtract a some what insignificant initial part of the second downward flat section (in Fig. 2 it is shown by a dash-line preceding this second, plateau section), using formulae (2) and (3) for this task:

$$
m_{(n+1) \ldots \infty}=m_{1 \ldots \infty}-m_{1 \ldots n}=A \frac{\exp (-k n)}{\exp k-1},
$$

i.e. $m_{1 \ldots n}$ here (in contrast with the aforementioned analogous symbol) is the formal isolated mass which should have belonged to the second (flat) section if that section had been the only one and let us repeat again, had begun with $n=1$, but not e.g. with $n=5$ (Fig. 5b). Herewith, empirical parameters $A$ and $k$ are the parameters of this flat section.

Then, for the given case of magnetic control of ferroimpurities in feldspar, the calculating formula (5) takes the following view:

$$
m_{1 \ldots \infty}=m_{1 \ldots n}+A \frac{\exp (-k n)}{\exp k-1} .
$$

This formula allows determining actual content of ferroimpurities (both extracted and residual ones) in the feldspar sample, and naturally, in the samples of likewise media (as is seen in Fig. 6, a dependence similar in view is observed
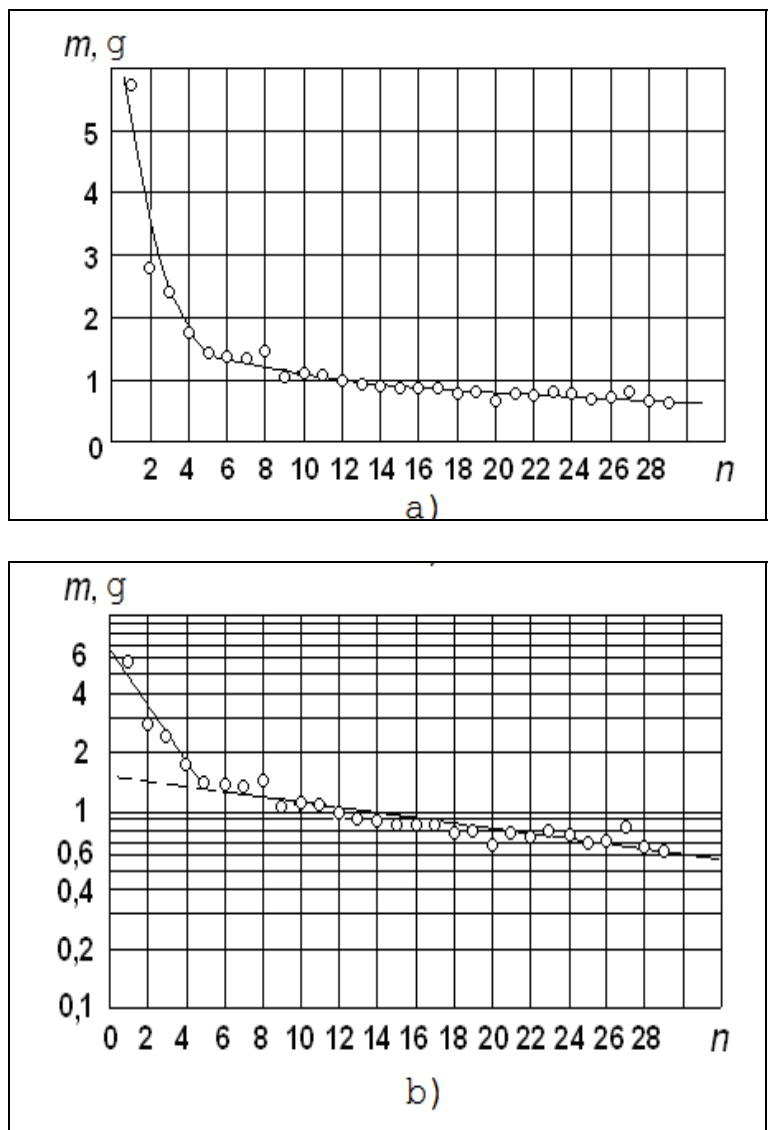

Figure 6. The same data as in Fig. 1 but for molding sand mixture in foundry production.

at magnetic control of ferroimpurities in sand and clay moulding mixtures for foundry production). As the research manifests, these quite peculiar media (feldspar, moulding mixes etc.) feature ferroparticles with a wide array of granularity, i.e. relatively 'easily-removed' and 'hard-to-remove' particles which eventually determine the 'piecewise-linear' view of $m, n$-dependences.

\section{Conclusion}

In this paper, it has been submitted to the literature that magnetic control of ferroimpurities of various granular and liquid media presupposes such as performing multiple operations of magnetic separating ferroimpurities from the sample of the medium analysed, obtaining a discrete dependence of ferroimpurities masses removed from the medium analysed on the sequence number of the magnetophoretic operation, functional legalisation of this dependence, employing this functional dependence for developing calculating formulae which allow defining total ag- 
gregate mass (including residual one) of ferroimpurities in a sample of given volume and/or mass have been observed. The method considered in the manuscript has been tested in such media as flour, grounded tealeaf, quartz sand, petrol, engine oil, feldspar, moulding sand mix for foundry production.

\section{Acknowledgement}

The research is conducted with financial support from RFFI within the frameworks of research project № 16-38-60034 mol_a_dk, the RF Ministry of Education and Science by the State Assignment in scientific activities №9.1189.2014/K.

\section{References}

1. D. Norrgran, T. Shuttleworth, G. Rasmussen. Updated magnetic separation techniques to improve grinding circuit efficiency, Minerals Engineering, 17 (2004).

2. P. Todd, R.P.Cooper, J.F. Dooyle, S. Dunn, J. Vellinger, M.S. Deuser, Multistage magnetic particle separator, Journal of Magnetism and Magnetic Materials, 225 (2001).

3. V. Zezulka, P. Straka, P. Mucha. A magnetic filter with permanent magnets on the basis of rare earth, Journal of Magnetism and Magnetic Materials, 268 (2004).

4. D. Norrgran. Magnetic filtration: producing fine high-purity feedstocks, Filtration and Separation, 45 (6), (2008).

5. J.M.D. Coey. Permanent magnet applications, Journal of Magnetism and Magnetic Materials, 248 (2002).

6. S. Nedelcu, J.H. Watson. Magnetic separator with transversally magnetized disk permanent magnets, Mineral Engineering, 15 (2002).

7. S. Sharma, A. Gaur, U. Singh, V. K. Katiyar. Modeling and Simulation of Magnetic Nanoparticles Transport in a Channel for Magnetic Drug Targeting,
Proceedings of the 12th International Conference on Heat Transfer, Thermal Engineering and Environment (HTE '14).

8. O. Hidehiko, M. Kazunari, O. Takeshi, R. Evan, W. Hiroshi. Computational Fluid Dynamics Simulation of High Gradient Magnetic Separation, Separation Science and Technology, 40 (2005).

9. J.G. Rayner, T.J. Napier-Munn. A mathematical model of concentrate solids content for the wet drum magnetic separator, International Journal of Mineral Processing, 70 (2003).

10. J. Svoboda. A realistic description of the process of high-gradient magnetic separation, Minerals Engineering, 14 (2001).

11. V.Murariu, J.Svoboda, P.Sergeant. The modeling of the separation process in a ferrohydrostatic separator, Minerals Engineering, 18 (2005).

12. K.Nandy, S.Chaudhuri, R.Ganguly, I.K.Puri. Analytical model for the magnetophoretic capture of magnetic microspheres in microfluidic devices, Journal of Magnetism and Magnetic Materials, 320 (2008).

13. R. Goleman. Macroscopic model of particles' capture by the elliptic crosssection collector in magnetic separator, Journal of Magnetism and Magnetic Materials, 272-276, (2004).

14. Patent 4492921 US. Method of Determining the Quantity of Solid Fraction of Ferromagnetic Matter in a Fluid. Sandulyak A.V., Garaschenko V.I., Korkhov O.J. Published 1985.

15. A.A. Sandulyak, D.A. Sandulyak, A.E. Ablaeva, A.V. Sandulyak. A Phenomenological Model of Experimental and Computational Magnetic Control of Ferrous Impurities in Foodstuffs, Proceedings of the 4th International Conference on Agricultural Science, Biotechnology, Food and Animal Science (Dubai, ABIFA15). 